Review Article

\title{
A Review of the Methods for Predicting the Effective In-Plane Shear Modulus of Cross-Laminated Timber (CLT)
}

\author{
Mehsam Tanzim Khan $\mathbb{D}^{1},{ }^{1}$ Ying Hei Chui, ${ }^{1}$ and Dongsheng Huang ${ }^{2}$ \\ ${ }^{1}$ Department of Civil and Environmental Engineering, University of Alberta, Edmonton T6G 2R3, Canada \\ ${ }^{2}$ National Engineering Research Center of Biomaterials, Nanjing Forestry University, Nanjing 210037, China \\ Correspondence should be addressed to Mehsam Tanzim Khan; mehsamta@ualberta.ca
}

Received 12 November 2020; Accepted 14 June 2021; Published 24 June 2021

Academic Editor: Daniele Baraldi

Copyright ( $) 2021$ Mehsam Tanzim Khan et al. This is an open access article distributed under the Creative Commons Attribution License, which permits unrestricted use, distribution, and reproduction in any medium, provided the original work is properly cited.

\begin{abstract}
Cross-laminated timber (CLT) is a type of engineered wood product that offers both high in-plane and out-of-plane load-bearing capacity. It is slowly becoming an alternative material for building high-rise structures. However, there is no current standard or regulation for determining the shear modulus of CLT under in-plane loading condition, which is a very important property for its use as structural members. Few methods have been proposed over the last decade to determine the in-plane shear modulus of CLT. Almost all of the methods proposed until now have their strengths and weaknesses. In this paper, some of the prominent methods for determining the in-plane shear modulus of CLT are described and analysed. The descriptions along with the critical discussions will facilitate a better understanding and might pave the way to further enhancements of the method(s) to determine the in-plane shear modulus of CLT.
\end{abstract}

\section{Introduction}

Most of the traditional timber structures have been built based on the light-wood framework system, which is an assembly of regularly spaced dimension lumber members that are fastened together to create structural components. Due to the re-emergence of global interest in timber structures and the introduction of a relatively new category of engineered wood products called mass timber panel (MTP), it has now become possible to construct more complex and long-span timber structures. This type of construction utilizing MTPs is termed mass timber construction. Mass timber construction is making headway in the construction industry in North America and some European countries. Use of mass timber panel can revolutionize the timber construction industry as it opens the door to "building tall with structural wood."

Cross-laminated timber (CLT) is a relatively new engineered wood product that began to be commercially produced in the mid-1990s and is categorized as a type of MTP. For a long time, the utilisation of CLT was limited to some
German-speaking countries, where the product is known as Brettsperrholz (BSP) [1]. The use of CLT is increasing because of its benefits compared to concrete and steel [2]. CLT usually consists of an odd number of layers $(3,5,7$, or 9 are the most common) of orthogonally glued timber boards/laminates. Each layer consists of adjacent boards which may or may not be edge-glued on the narrow face, depending on the manufacturer and the product type. CLT offers high load-carrying capacity under both in-plane and out-of-plane loading conditions due to the orthogonal arrangement of the laminates. For this reason, it can be used as both wall or edge-wise bending beam elements under in-plane loading condition and floor diaphragm panels under out-of-plane loading condition. Research has also proved that CLT is highly energy-efficient, and it has a high capacity for storing moisture and thermal energy. A $100 \mathrm{~mm}$ thick CLT has a very small thermal transmittance value of around $1.0 \mathrm{~W} / \mathrm{m}^{2}-\mathrm{K}$, which is a measure of resistance to the movement of heat, and an equivalent air layer thickness of SD $2-5 \mathrm{~m}$, which is a measure of resistance to moisture diffusion compared to a meter of air's resistance to moisture diffusion $[3,4]$. 
When CLT is used under in-plane loading condition as beam, lintel, or wall panel, one major benefit of CLT is its high tensile strength perpendicular to the beam axis, which makes it less susceptible to crack formation in the perpendicular to the grain direction of the laminates of each layer [5]. CLT has been reported to have higher in-plane shear strength than other similar engineered wood products like glulam [1]. In order to get the full benefit of CLT panels under in-plane loading condition, detailed knowledge and investigation of all the relevant mechanical properties are indispensable. In-plane elastic properties (shear modulus $(G)$ and bending modulus $(E)$, as well as shear stiffness (GA) and bending stiffness (EI), are some of the vital parameters of CLT for its use in structural members [6]. Aside from their use in ultimate limit state (ULS) design, to meet the requirements of serviceability limit states (SLSs), the design checks require elastic properties as input parameters [7]. Therefore, it is necessary to accurately characterize the elastic properties and stiffness of CLT under in-plane loading condition for their structural application as beams, lintels, or wall elements.

Several recent studies have attempted to predict the inplane shear modulus $(G)$ of CLT panels by different methods. Among these, the methods developed by Brandner et al. [6], Flaig and Blaß [5], and Bogensperger et al. [1] have gained prominence. Brandner et al. [6] used the constitutive equations of the laminates and developed a semiempirical method to predict the in-plane shear modulus of CLT. Flaig and Blaß [5] treated CLT under in-plane loading condition as a transversely loaded beam. Following the beam analogy, they formulated an analytical model to predict the in-plane shear modulus of CLT. Bogensperger et al. [1] employed finite element analysis to develop a model for predicting the inplane shear modulus of CLT. Among other studies, the studies conducted by Turesson et al. [8] and Andreolli et al. [9] are noteworthy. Turesson et al. [8] proposed some reduction factors from finite element analysis for a range of configurations of CLT which, when multiplied by the in-plane shear modulus of the laminates of the CLT, give the value of the effective in-plane shear modulus of the whole CLT. Andreolli et al. [9] subjected square-shaped CLT specimens to diagonal compression tests with an ad-hoc test setup and experimentally measured the shear modulus of CLT.

Though there have been a few investigations on the inplane shear modulus of CLT, a comprehensive analysis and comparison of the available methods to determine the shear modulus is not available to date. Recognizing this gap in the body of knowledge, this paper will focus on a detailed comparative analysis of the available methods for the determination of the shear modulus value, along with some recommendations. Thus, this paper can act as an exhaustive summary of almost all the contemporary methods for determining the inplane shear modulus of CLT and will facilitate further research on the in-plane shear modulus of CLT.

\section{Discussion}

As discussed in the previous section, several methods for the determination of the in-plane shear modulus of CLT as proposed by different researchers are prevalent. The methods were only briefly introduced in the previous section. In this section, the methods proposed by Brandner et al. [6], Flaig and Blaß [5], Bogensperger et al. [1], Turesson et al. [8] and Andreolli et al. [9] will be discussed in detail. All of these methods emphasize on determining the shear modulus of a non-edge-glued CLT. In edge-glued CLT, since the adjacent laminates of each layer are bonded, the CLT deforms as a whole continuous unit under shear stress and behaves similar to a piece of lumber. Thus, the shear modulus of edge-glued CLT is commonly taken to be equivalent to the shear modulus of the lumber forming the CLT [8].

2.1. Brandner Method. Brandner et al. [6] adopted the test configuration of Kreuzinger and Sieder [10] and proposed a method to determine the shear modulus of CLT from a simple compression test conducted on a differential CLT section. Instead of testing full scale CLT panels, Brandner et al. [6] opted for smaller CLT specimens to realize a continuous load path in the test samples, to achieve pure shear failure mode, and to minimize the test implementation cost.

In the test configuration proposed by Kreuzinger and Sieder [10], a rectangular section is cut out at $45^{\circ}$ angle to the direction of the outer longitudinal layers of the CLT. The rectangular section has a length of $1500 \mathrm{~mm}$ and a width of $500 \mathrm{~mm}$. This section is then subjected to a compressive load $(F)$ as exhibited in Figure 1.

In Figure 1, $x$ and $y$ directions are the global horizontal and vertical directions of the system, respectively (the global coordinate system), and $x_{M}$ and $y_{M}$ are the directions of the fibres of the outer longitudinal layers and the transverse (cross) layers of the CLT, respectively (the local/material coordinate system). The local coordinate system is rotated counter-clockwise by an angle of $45^{\circ}$ with respect to the global coordinate system. Under the action of the compressive load $(F)$, the stress state of the CLT section cut out at $45^{\circ}$ constitutes pure shear $\left(\tau_{x M y M}\right)$ combined with pure compression $\left(\sigma_{y M}\right)$ in the local coordinate system. In the global coordinate system, only the vertical stress component $\left(\sigma_{y}\right)$ is created on the CLT section. Using stress-strain relationships from the plate theory (in-plane stresses), the constitutive equations for the material of the CLT in the local coordinate system are given as

$$
\left(\begin{array}{c}
\varepsilon_{x M} \\
\varepsilon_{y M} \\
\gamma_{x M y M}
\end{array}\right)=\left(\begin{array}{ccc}
\frac{1}{E_{x M}} & 0 & 0 \\
0 & \frac{1}{E_{y M}} & 0 \\
0 & 0 & \frac{1}{G_{x M y M}}
\end{array}\right)\left(\begin{array}{c}
\sigma_{x M} \\
\sigma_{y M} \\
\tau_{x M y M}
\end{array}\right),
$$

where $\varepsilon_{x M}, \varepsilon_{y M}$, and $\gamma_{x M y M}$ are the strain components and $\sigma_{x M}, \sigma_{y M}$, and $\tau_{x M y M}$ are the stress components. $E_{x M}$ is the weighted modulus of elasticity in the $x_{M}$ direction, and $E_{y M}$ is the weighted modulus of elasticity in the $y_{M}$ direction. 


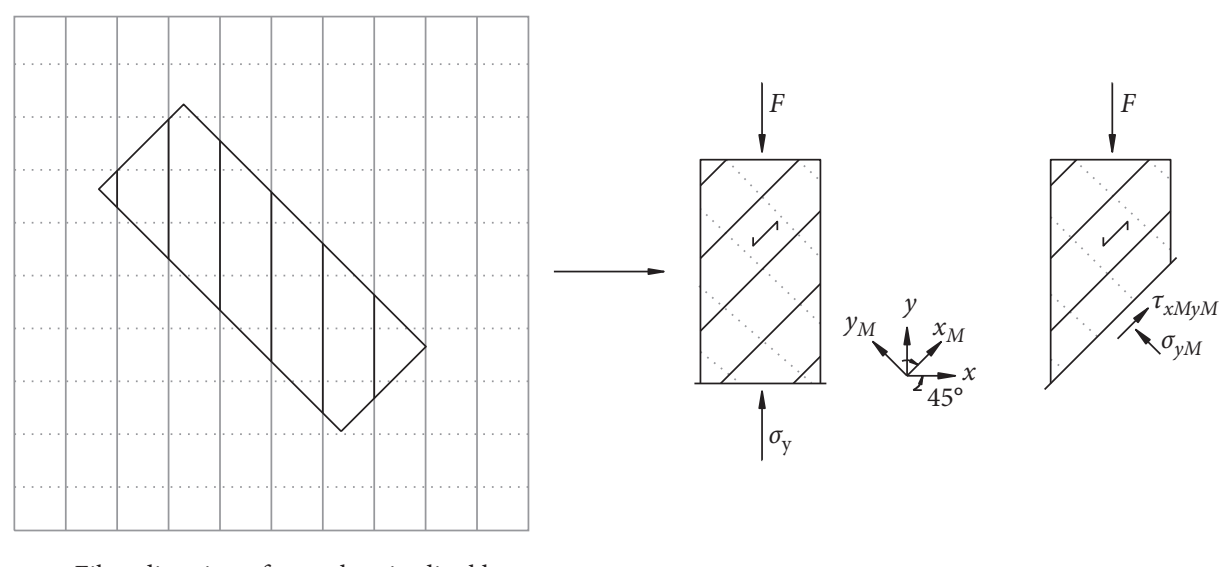

FIgURE 1: Internal stresses and external loading of the rectangular CLT cut out at $45^{\circ}$ angle from larger CLT panels according to Brandner et al. [6].

$G_{x M y M}$ is simply the shear modulus of the CLT section. $E_{x M}$ and $E_{y M}$ can be determined as follows by the method of weighted average:

$$
\begin{aligned}
& E_{x M}=\frac{\sum t_{x M} E_{0}+\sum t_{y M} E_{90}}{t_{\mathrm{CLT}}} ; \\
& E_{y M}=\frac{\sum t_{y M} E_{0}+\sum t_{x M} E_{90}}{t_{\mathrm{CLT}}},
\end{aligned}
$$

where $t_{x M}$ is the thickness of each longitudinal layer, $t_{y M}$ is the thickness of each transverse layer, $E_{0}$ is the modulus of elasticity of each layer parallel to the grain, $E_{90}$ is the modulus of elasticity of each layer perpendicular to the grain, and $t_{\text {CLT }}$ is the total thickness of CLT.

$$
S_{x M y M}=\left(\begin{array}{ccc}
\left(1 / E_{x M}\right) & 0 & 0 \\
0 & \left(1 / E_{y M}\right) & 0 \\
0 & 0 & \left(1 / G_{x M y M}\right)
\end{array}\right) \text { in (2) is }
$$

the flexibility matrix in the local coordinate system which can be transformed to the global coordinate system $\left(S_{x y}\right)$ using the following transformation:

$$
S_{x y}=T S_{x M y M} T^{T}
$$

where

$$
T=\left(\begin{array}{ccc}
\cos ^{2} \alpha & \sin ^{2} \alpha & -\sin \alpha \cos \alpha \\
\sin ^{2} \alpha & \cos ^{2} \alpha & \sin \alpha \cos \alpha \\
2 \sin \alpha \cos \alpha & -2 \sin \alpha \cos \alpha & \cos ^{2} \alpha-\sin ^{2} \alpha
\end{array}\right)
$$

where $\alpha$ is the angle between the local and the global coordinate systems, which in this case is $45^{\circ}$. For $\alpha=45^{\circ}$, the flexibility matrix in the global coordinate system is given by

$$
S_{x y}=\left(\begin{array}{ccc}
\frac{0.25}{E_{x M}}+\frac{0.25}{E_{y M}}+\frac{0.25}{G_{x M y M}} \frac{0.25}{E_{x M}}+\frac{0.25}{E_{y M}}-\frac{0.25}{G_{x M y M}} \frac{0.5}{E_{x M}}-\frac{0.5}{E_{y M}} \\
\frac{0.25}{E_{x M}}+\frac{0.25}{E_{y M}}-\frac{0.25}{G_{x M y M}} \frac{0.25}{E_{x M}}+\frac{0.25}{E_{y M}}+\frac{0.25}{G_{x M y M}} \frac{0.5}{E_{x M}}-\frac{0.5}{E_{y M}} \\
\frac{0.5}{E_{x M}}-\frac{0.5}{E_{y M}} & \frac{0.5}{E_{x M}}-\frac{0.5}{E_{y M}} & \frac{1}{E_{x M}}+\frac{1}{E_{y M}}
\end{array}\right) .
$$

Thus, the flexibility matrix given in (5) is the flexibility of the CLT section in the global directions with the properties transformed from the local material directions to the global directions. Now, in the global coordinate system, the constitutive equations for the material of the CLT are given as 


$$
\begin{aligned}
\left(\begin{array}{c}
\varepsilon_{x} \\
\varepsilon_{y} \\
\gamma_{x y}
\end{array}\right) & =\left(\begin{array}{ccc}
\frac{1}{E_{x}} & 0 & 0 \\
0 & \frac{1}{E_{y}} & 0 \\
0 & 0 & \frac{1}{G_{x y}}
\end{array}\right)\left(\begin{array}{c}
\sigma_{x} \\
\sigma_{y} \\
\tau_{x y}
\end{array}\right) \\
& =\left[\begin{array}{c}
\sigma_{x y} \\
\sigma_{x y}
\end{array}\right),
\end{aligned}
$$

where, similar to (1), $\varepsilon_{x}, \varepsilon_{y}$, and $\gamma_{x y}$ are the strain components and $\sigma_{x}, \sigma_{y}$, and $\tau_{x y}$ are the stress components in the global directions. $E_{x}$ is the modulus of elasticity in the $x$ direction, and $E_{y}$ is the modulus of elasticity in the $y$ direction. $G_{x y}$ is the shear modulus of the CLT section $\left(G_{x M y M}\right.$ and $G_{x y}$ are synonymous). The flexibility matrix $S_{x y}$ given in (6) is equivalent to that given by (5). The only difference between them is as follows: one is derived from the local material directions and transformed into the global directions, while the other is given directly in the global axes.

From the direction of load application on the rectangular section, it can be observed that $\sigma_{x}=0$ and $\tau_{x y}=0$ since only vertical compressive load is acting on the section. Thus, under the vertical compressive load, a discrete stress state $\sigma_{y}$ with an associated strain $\varepsilon_{y}$ is formed which follows the simple constitutive relation $\sigma_{y}=E_{y} \varepsilon_{y} . \sigma_{y}$ is the stress acting in the vertical direction on the CLT section, and $\varepsilon_{y}$ is the associated strain. From the vertical load-deflection behaviour of the CLT section, the modulus of elasticity in the vertical direction, $E_{y}$ can be determined. Now, considering the $(2,2)$ element of the flexibility matrix given in equations (5) and (6), and their equivalency, the following relationship holds:

$$
\frac{1}{E_{y}}=\frac{0.25}{E_{x M}}+\frac{0.25}{E_{y M}}+\frac{0.25}{G_{x M y M}} .
$$

Rearranging (7), the shear modulus can be determined using

$$
G_{x M y M}=\frac{1}{\left(\left(4 / E_{y}\right)-\left(1 / E_{x M}\right)-\left(1 / E_{y M}\right)\right)},
$$

where $E_{y}$ is determined from vertical load-deflection behaviour of the CLT section and $E_{x M}$ and $E_{y M}$ are estimated from the material properties of the laminates that form the CLT. Equation (8) represents the final form of Brandner et al.'s [6] model to predict the shear modulus of CLT. The test setup used by Brandner et al. [6] is shown in Figure 2. The CLT section is placed in between the machine actuator head at the top and the support (machine base) at the bottom. Measurement crosses are placed concentrically on the specimen to measure both the vertical and the horizontal deflection.

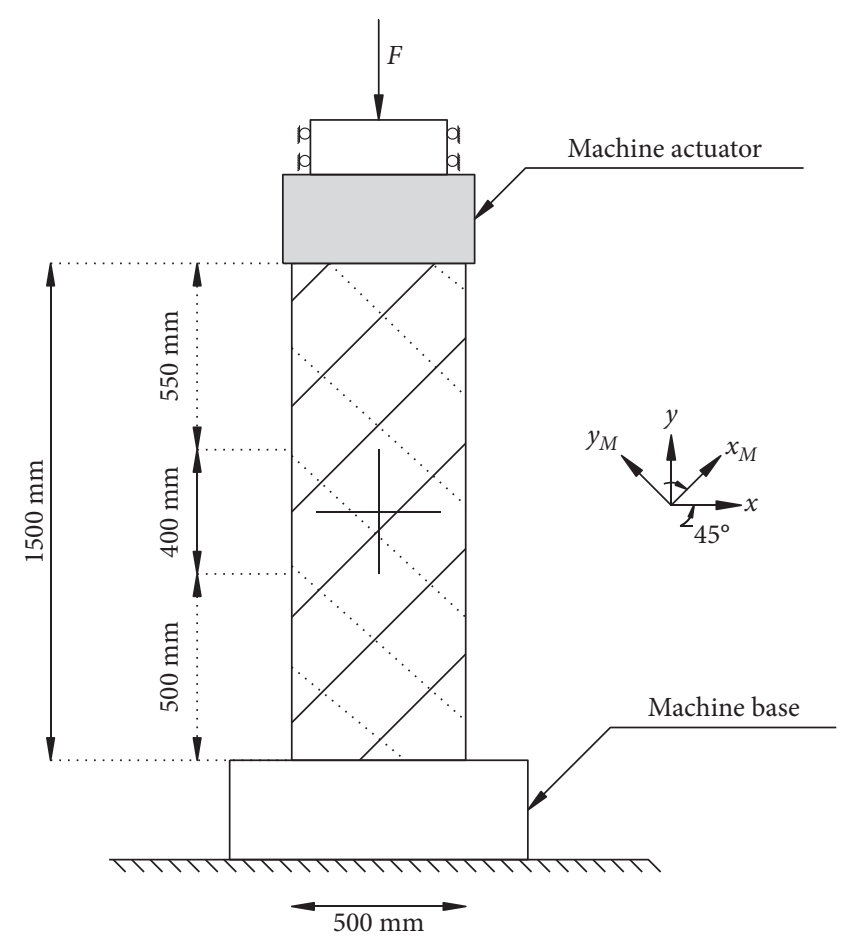

Figure 2: Test setup used by Brandner et al. [6]. The specimen is placed between the machine base and the machine actuator.

The test configuration adopted by Brandner et al. [6] was motivated by noting the difficulties faced in determining shear properties on a full-scale CLT diaphragm, as had been performed by Andreolli et al. [9] and Bogensperger et al. [1]. The simple compression test can be conducted in almost any laboratory that has a compression test machine without the need for any complicated instrumentation. The intended and assumed failure mode in this configuration is the gross-shear failure mode, which is initiated by the longitudinal shearing failure (shear failure parallel to the grain) in all the layers of the CLT considering the gross cross-section of CLT. The in-plane deformation (both vertical and horizontal) of the CLT specimen under compressive load was determined on both side faces using centrically placed measurement crosses with a gauge length of $400 \mathrm{~mm}$. The height-to-width ratio of the specimen used in Brandner et al. [6] tests was $1500 \mathrm{~mm} /$ $500 \mathrm{~mm}=3 / 1$. Through finite element analysis, it was verified that this height-to-width ratio of the specimen did not lead to any stress concentrations at the middle of the specimen. Also, the middle of the specimen was under a state of constant shear stress. Thus, deformation measurements were taken in this middle region of the specimen as exhibited in Figure 2.

Shear modulus values were experimentally determined by Brandner et al. [6] using two approaches, (a) by using (8), with the value of $E_{y}$ measured from the load-deformation data in the vertical direction of the test configuration of Figure 2, and standardized moduli of elasticity, $E_{0}$ and $E_{90}$ according to the stress class of the lumbers of each layer of the CLT; (b) with the approach standardized in EN 408 [11]. The second approach involved measuring the local shear deformations of a specimen in a bending test. Details of this approach can be found in the standard EN 408 [11]. The 
shear modulus values using these two approaches differed by about $10 \%$, the latter approach yielding higher values. Brandner et al. [6] noted the higher deflection in the vertical direction compared to the horizontal direction in the compression test setup as a possible reason for the lower value given by the first approach. Among the two approaches, the first approach led to higher variability in the shear modulus values.

From (1), it can be observed that the off-diagonal elements, which are related to Poisson's ratio, have been ignored. Furthermore, in estimating $E_{x M}$ and $E_{y M}, E_{0}$ might be considered as the modulus of elasticity of each layer parallel to the grain direction of the laminates of the layer and $E_{90}$ is the modulus of elasticity of each layer perpendicular to the grain direction of the laminates of that layer. While assuming $E_{0}$ of each layer to be equal to the $E_{0}$ of the laminates of the layer might be appropriate, assuming $E_{90}$ of each layer to be equal to the $E_{90}$ of the laminates might be an overestimation. The rationale behind this is, for each layer, the continuity of the laminates which is maintained when stresses parallel to the grain (fibre) direction are considered and the laminates can be considered to be fully effective in resisting the stresses. On the other hand, when stresses perpendicular to the grain (fibre) direction are considered, the continuity of laminate is disrupted at the boundary between two adjacent laminates due to the presence of edgegaps and inadequate bonding (for CLT without edge-gluing or narrow face bonding), and the full capacity of the laminates might not be effective in resisting the stresses. Thus, assuming the modulus of elasticity of the layer perpendicular to the grain direction of the laminates equal to the modulus of elasticity of the laminate material perpendicular to the grain may lead to an overestimation of the stiffness property. Also, the specimen size adopted in the test configuration is still fairly sizeable, which led to heavy specimens and the need to use lifting equipment in the test laboratory.

2.2. Flaig and Blaß Method. According to Flaig and Blaß [5], when CLT is subjected to in-plane transversal forces, the overall shear deformation in the CLT can be considered to be the superposition of two components: (1) shear deformation within the laminates of the CLT and (2) rotational and translational deformation of the crossing areas between the laminates of the longitudinal and the transversal layers. The first component arises from the shear strain within the laminates of the CLT, and it is equivalent to the shear deformation in wood material. This is the only component of shear deformation in an edge-glued CLT (CLT with narrow face bonding). The second component arises from the mutual displacement and rotation that occur in the crossing area between the laminates of the longitudinal and the transversal layers in a CLT without narrow face bonding [12]. The glued connection between the longitudinal and transverse layers (crossing area) of the CLT can be considered as rigid; however, the wood fibres at the vicinity of the adhesive layer in the crossing areas can undergo significant deformation under transverse loads. This leads to the mutual displacement and rotation that occur in the crossing area, or more precisely, at the vicinity of the crossing area. Since shear deformation within the laminate is the only component of shear deformation in an edge-glued CLT, the shear modulus of an edge-glued CLT can be considered to be comparable to that of a solid lumber and it was not considered in the Flaig and Blaß [5] study.

The two components of shear deformation in a differential CLT section that arise due to the action of the shear force Ton all faces are illustrated in Figure 3. The strain $\gamma_{S}$ in the left picture is the first component of the shear deformation, and $\gamma_{C A}$ in the right picture is the second component of the shear deformation. Some implicit assumptions of the Flaig and Blaß [5] model are as follows: (1) the CLT is loaded on edge as a beam; (2) the transverse loads acting on the CLT beam are perpendicular to the direction of the grain of the outer longitudinal layers, and (3) the direction of the grain of the outer longitudinal layers is aligned with the beam axis.

Along the length or width of the CLT, in sections which coincide with gaps between adjacent laminates of the same layer, the shear forces have to be transferred to the subsequent layer in the CLT thickness direction through the crossing areas. This leads to the development of shear stress in the crossing areas. The second component of the overall shear deformation arises from the stress acting in the crossing areas. This second component of the overall shear deformation can again be broken down into two mechanisms, translational and the rotational. The translational deformation $\left(\gamma_{y x}\right)$ originates from unidirectional shear stresses acting parallel to the beam axis (Figure 4(a)), while the rotational deformation $\left(\gamma_{\text {tor }}\right)$ is caused by torsion as shown in Figure 4(b).

The strain components are given by the following relationships:

$$
\begin{aligned}
\gamma_{y x} & =\frac{2 \tau_{y x}}{K w(m-1)} \\
& =\frac{12 V}{K w^{3} m^{3} n_{C A}}, \\
\gamma_{\text {tor }} & =\frac{2 \tau_{\text {tor }}}{K w} \\
& =\frac{6 V}{K w^{3} n_{C A}}\left(\frac{1}{m}-\frac{1}{m^{3}}\right) .
\end{aligned}
$$

In the above equations, $\tau_{y x}$ is the shear stress acting parallel to the beam axis of the CLT and it is caused by the variation of the bending moment along the beam axis. If the CLT does not have narrow face bonding, the differential normal forces that arise from the change of the bending moment along the beam axis have to be transferred through the crossing areas between the longitudinal and the transverse layers. This is the source of the shear stress $\tau_{y x}$. $\tau_{t o r}$ is the torsional shear stress which arises from the eccentricity between the centre lines of the laminates of the longitudinal layers with respect to the central plane of the CLT beam (the horizontal plane through the midheight of the CLT beam). The torsional shear stress is assumed to be constant within the beam thickness and uniformly distributed within the 


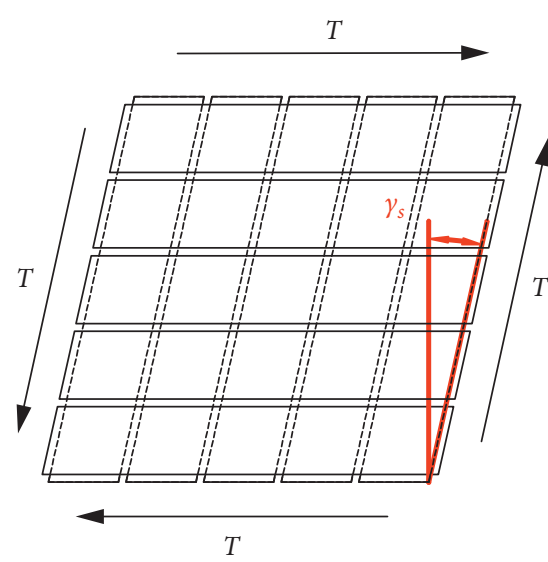

(a)

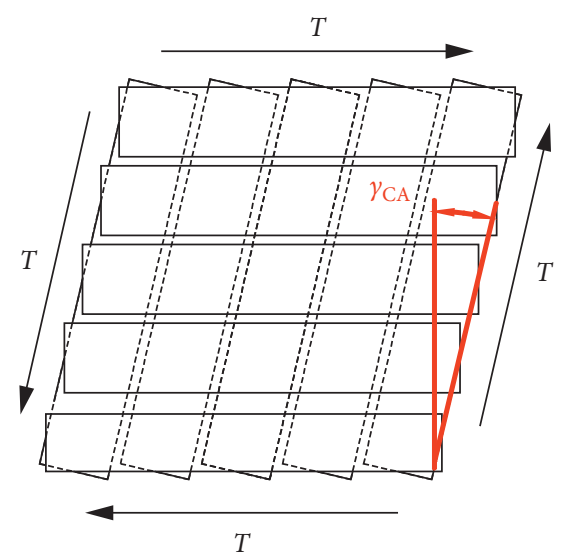

(b)

Figure 3: The two components of the overall shear deformation: (a) shear deformation within the laminates; (b) rotational and translational deformation in the crossing areas [12].

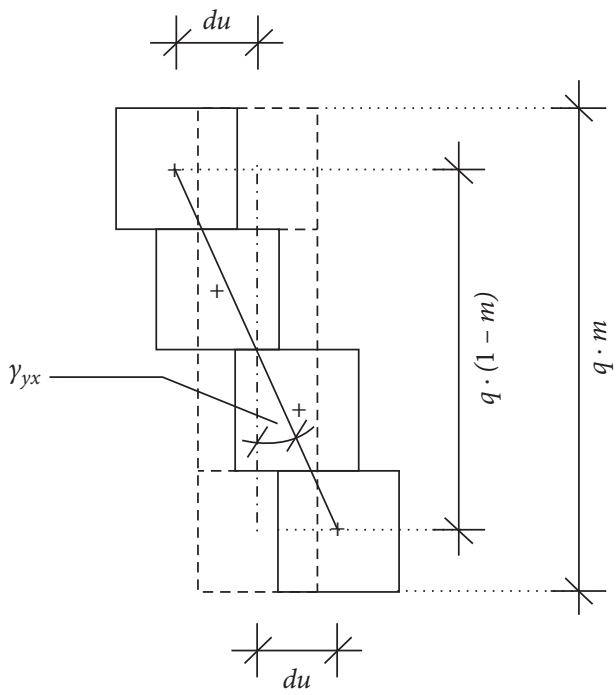

(a)
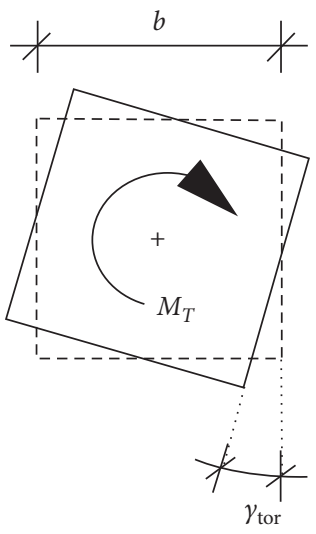

(b)

FIgURE 4: Shear strain components in the crossing areas due to (a) the translational deformation mechanism and (b) the rotational deformation mechanism [5].

beam height. The latter assumption is only possible if the laminates of the transverse layers remain straight (do not undergo any rotation) in the deformed beam. This torsional shear stress leads to rolling shear stresses in the laminates. $K$ is the slip modulus, a measure of the stiffness of the crossing areas with the unit of force/(length) ${ }^{3}$. In (9) and (10), no distinction has been made between the stiffness of the crossing areas under the two separate mechanisms (e.g., slip modulus and torsional modulus). The same $K$ value is used as the measure of stiffness under both mechanisms. $w$ is the width of all the laminates, which is taken as constant for all the layers. Information on CLT with laminates of different widths in the longitudinal and transverse layers can be found in Blaß and Flaig [13]. $m$ is the number of longitudinal laminates within the beam height direction. $V$ is the shear force acting on a section of the CLT beam, and $n_{C A}$ is the number of glue lines between the longitudinal and transverse layers in the beam width direction.

From (9) and (10), it can be observed that the number of laminates in the longitudinal layers have a pronounced effect on the shear deformations. However, in CLT beams having a large number of laminates in the longitudinal layers, the last term in (10) becomes very small and can be ignored.

For CLT with a rectangular cross-section, the constitutive equation for the crossing area is

$$
\tau=\left(\gamma_{y x}+\gamma_{\text {tor }}\right) G_{\text {eff }, C A},
$$

where $G_{\text {eff, } C A}$ is the shear modulus of the crossing area and $\tau$ is the shear stress acting in the crossing area. To estimate this shear stress from the shear force acting in the gross crosssection (considering the whole thickness and width of the CLT), a shear correction factor has to be used: 


$$
\tau=k_{\text {shear }} \frac{V}{A_{\text {gross }}},
$$

where $A_{\text {gross }}$ is the gross cross-sectional area and $k_{\text {shear }}$ is the shear correction factor, assumed to be $5 / 6$ by Flaig and Blaß [5] which is a value commonly used for rectangular crosssections [14], and $V$ is the shear force acting in the whole CLT gross cross-section. Thus, the shear modulus of the crossing area is given by

$$
\begin{aligned}
G_{\text {eff }, C A} & =\frac{6 V}{5 A_{\text {gross }}\left(\gamma_{y x}+\gamma_{\text {tor }}\right)} \\
& =\frac{K w^{2} m^{2} n_{C A}}{5 t_{\text {CLT }}\left(m^{2}+1\right)},
\end{aligned}
$$

where $t_{\text {CLT }}$ is the total thickness of the CLT beam and the terms on the right side of (12) have been derived by substituting the values of $\gamma_{y x}$ and $\gamma_{\text {tor }}$ from equations (9) and (10). Finally, the shear deformations in the laminates and the deformation of the crossing areas are superimposed by the following formalism:

$$
\begin{aligned}
\gamma_{\mathrm{eff}, \mathrm{CLT}} & =\gamma_{\mathrm{eff}, C A}+\gamma_{\mathrm{lam}}, \\
\gamma_{\mathrm{eff}, C A} & =\gamma_{y x}+\gamma_{\mathrm{tor}}, \\
\tau & =\gamma_{\mathrm{eff}, C A} G_{\mathrm{eff}, C A}=\gamma_{\mathrm{lam}} G_{\mathrm{lam}} \\
& =\gamma_{\mathrm{eff}, \mathrm{CLT}} G_{\mathrm{eff}, \mathrm{CLT}} \\
& =\left(\gamma_{\mathrm{lam}}+\gamma_{\mathrm{eff}, C A}\right) G_{\mathrm{eff}, \mathrm{CLT}}, \\
\tau & =\left(\frac{\tau}{G_{\mathrm{lam}}}+\frac{\tau}{G_{\mathrm{eff}, C A}}\right) G_{\mathrm{eff}, \mathrm{CLT}}, \\
G_{\mathrm{eff}, \mathrm{CLT}} & =\left(\frac{1}{G_{\mathrm{lam}}}+\frac{1}{G_{\mathrm{eff}, C A}}\right)^{-1},
\end{aligned}
$$

where $\gamma_{\text {eff,CLT }}$ is the overall shear deformation in the CLT beam, $\gamma_{\text {eff,CA }}$ is the total shear deformation of the crossing areas due to the two mechanisms, and $\gamma_{\text {lam }}$ is the shear deformation within the laminates. $\tau$ is the shear stress acting on the crossing area as before, and also in the laminates and the whole CLT. Thus, $\tau$ is the effective shear stress acting on the CLT. $G_{\text {lam }}$ is the mean shear modulus of the laminates (Flaig and Blaß [5] assumed all the laminates of the CLT to have the same shear modulus) of the CLT, and $G_{\text {eff,CLT }}$ is the effective shear modulus of the whole CLT. Equation (14) represents the final closed form solution of the Flaig and Blaß [5] model to predict the shear modulus of CLT.

The determination of the stiffness of the crossing areas (slip modulus, $K$ in (9) and (10)) is a challenge as discussed by Blaß and Flaig [13], and the results of the studies carried out by Blaß and Görlacher [15] and by Jöbstl et al. [16] have a significant discrepancy. Blaß and Flaig [13] carried out fourpoint bending tests on CLT beams. From the measured shear-free and apparent moduli of elasticity of the CLT beams, the effective shear modulus values of the CLT beams were evaluated. Using the effective shear modulus value, the slip modulus of a crossing area was determined using (13) and (14) and a laminate shear modulus of $690 \mathrm{~N} / \mathrm{mm}^{2}$, which is the characteristic value of the shear modulus of lumber of nominal strength class C24 according to EN 338 [17].

The shear modulus values tabulated in Flaig and Blaß [6] are determined the measured shear-free and apparent moduli of elasticity of the CLT beams under four-point bending tests. No ad hoc test setup for measurement of shear modulus was made. The problem with the four-point bending test is that the shear deformations under bending test might be very small depending on the span-to-depth ratio $(\mathrm{L} / \mathrm{h})$ of the CLT beam. Nevertheless, Flaig and Blaß [6] found that the shear modulus of CLT beam increases with the number of layers of CLT.

Though Flaig and Blaß [6] pointed out that the shear stress component $\tau_{y x}$ varies in the beam width direction for CLT in which the ratio of $t_{\text {long, } k} / n_{C A, k}$ ( $t_{\text {long, } k}$ is the thickness of the $k$ th longitudinal layer and $n_{C A, k}$ is the number of glue lines the $k$-th longitudinal layer shares with adjacent transverse layers) is not constant for all the longitudinal layers, it was assumed that this shear stress variation in the beam width direction can be ignored. Danielsson and Serrano [18] pointed out that, this assumption is inaccurate, and this shear stress component is significantly influenced by the lay-up parameters $t_{\text {long, } k} / t_{\text {net,long }}\left(t_{\text {net,long }}\right.$ is the sum of the thicknesses of the longitudinal layers), $n_{C A}$, and $n_{C A, k}$. For the calculation of shear stress component $\tau_{\text {tor }}$, Danielsson and Serrano [18] also noted that the assumption of equal torsional moments for all crossing areas in the beam height direction is inaccurate in the Flaig and Blaß [5] model. Danielsson and Serrano [18] found the torsional moments and shear forces to be significantly greater close to the centre line of the beam compared to the upper and lower parts of the beam. The findings of Danielsson and Serrano [18] might have implications on the method of effective shear modulus determination proposed by Flaig and $\mathrm{Blaß}[5]$.

2.3. Bogensperger Method. Bogensperger et al. [1] took advantage of the regular periodic geometric structure of CLT elements and modelled the smallest unit cell for mechanical treatment, called the "representative volume subelement (RVSE)." The unit cell which is repeated over the whole volume of the CLT is called the "representative volume element (RVE)." The RVE can be decomposed to even smaller building blocks called the "representative volume subelement (RVSE).” The representative volume subelement comprises of a cut out of two orthogonal laminates in two adjacent layers of CLT, usually each of them extending over half of the thickness of each layer. This further decomposition of RVE into RVSE is justified if an infinite number of layers of CLT are assumed. The RVSE is surrounded by two planes of symmetry on the two sides. The RVE and RVSE are shown in Figure 5.

From Figure 5, it can be observed that, if there are $n$ number of layers in CLT, the number of glue planes and thus the number of RVSE are $(n-1)$. Also, each RVSE is 


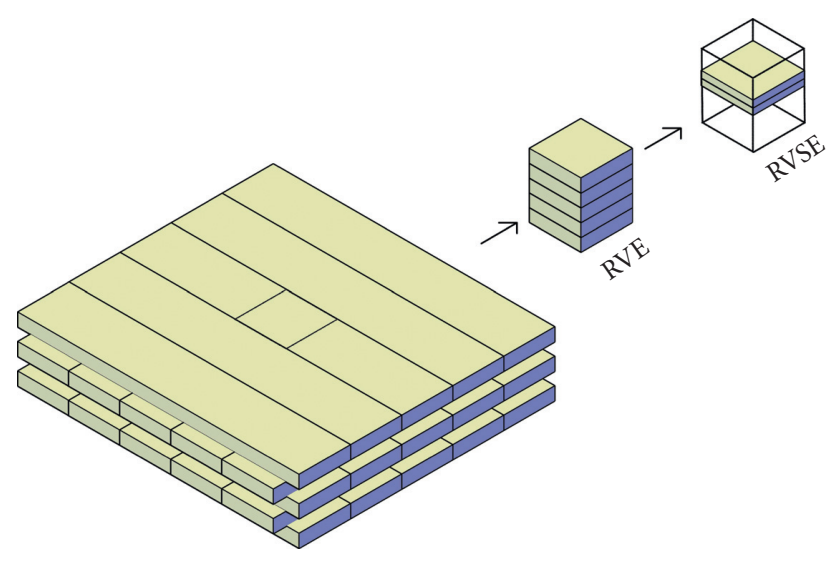

Figure 5: A full-sized CLT panel decomposed to an RVE and then subsequently to an RVSE.

composed of one glue plane and two orthogonal laminates on either side of the glue plane. Bogensperger et al. [1] assumed the thickness of the thinner of the two glued laminates to be the controlling thickness of the RVSE, as a conservative approach to calculation of the resulting shear stresses. However, the thickness of the outermost RVSEs in a CLT is equal to either twice the thickness of the outer laminate or the ordinary thickness of the inner laminate, whichever is less. To illustrate the thickness of the RVSE in detail, the RVSEs that constitute a 5-layer CLT are shown in Figure 6.

As the CLT in Figure 6 is a 5-layer CLT, it has 4 RVSEs. For the 4 RVSEs in Figure 6, the thickness of each RVSE is given in Table 1.

The overall thickness of all the RVSEs is equal to the sum of the thickness of all the RVSEs, which is always smaller than or equal to the overall geometric thickness of the CLT.

Under in-plane shear forces, shear stresses are generated in such a way that the narrow faces of the laminates of each layer remain stress-free. These narrow faces usually have glue lines in an edge-glued CLT while they lack glue lines in a non-edge-glued CLT. Shear stresses are transferred to the adjacent RVSEs at the vertical and horizontal pairs of planes of periodicity only. This means that shear stresses are not transferred to adjacent laminates of the same layer in a nonedge-glued CLT. The complete in-plane shear mechanism in an RVSE can be decomposed into two parts: (a) pure shear mechanism with full shear force transmission at the narrow faces of all boards (mechanism 1, Figure 7); (b) local stress redistribution caused by torsional moments acting on both sides of the gluing interface on the wide faces of the laminates (mechanism 2, Figure 8). These two mechanisms superimpose to create a stress-free condition at the narrow faces (Figure 9(a)). This fulfils the condition of no shear stress transmission to adjacent laminates of the same layer through the narrow faces.

The mechanism 1 corresponds to the state of uniform shear across the total thickness of the RVSE (Figure 9(b)). This state occurs in homogeneous CLTs with narrow face bonding (edge-gluing). Under this mechanism, full shear force is transmitted at the narrow faces of the laminates.

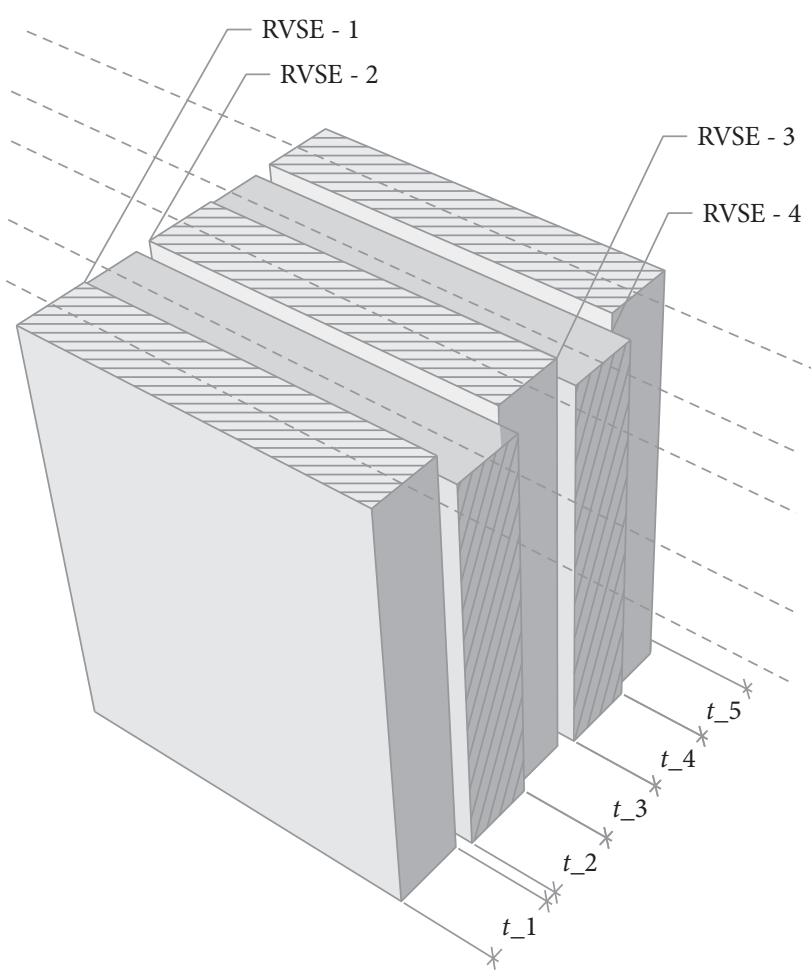

FIgURE 6: Idealized RVSE for a 5-layer CLT according to Bogensperger et al. [1].

TABLE 1: Idealized thicknesses of all the RVSEs for the CLT in Figure 6

\begin{tabular}{lc}
\hline RVSE designation & Thickness \\
\hline RVSE-1 & $\min \left(2 t_{1}, t_{2}\right)$ \\
RVSE-2 & $\min \left(t_{2}, t_{3}\right)$ \\
RVSE-3 & $\min \left(t_{3}, t_{4}\right)$ \\
RVSE-4 & $\min \left(t_{4}, 2 t_{5}\right)$ \\
\hline
\end{tabular}
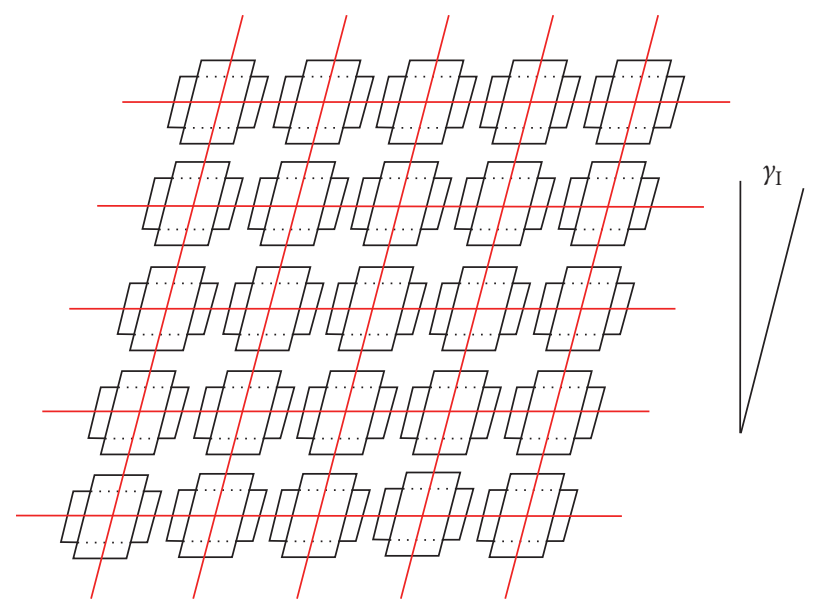

FIgURe 7: Pure shear mechanism, mechanism 1 [1].

Therefore, the relevant shear stiffness under mechanism 1 is equivalent to the mean shear modulus of the laminates parallel to the grain $\left(\mathrm{G}_{\mathrm{lam}}\right)$. Mechanism 2 corresponds to a complicated state of torsion-like behaviour in the 


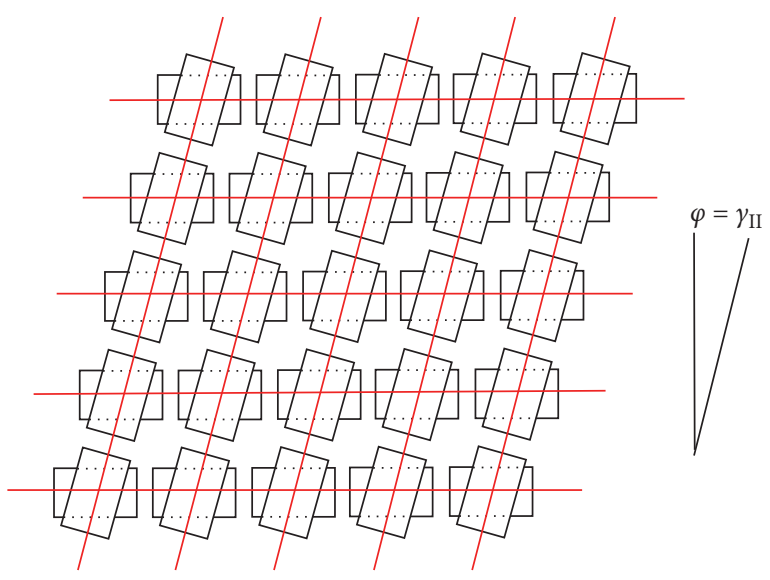

FIgURE 8: Local stress redistribution due to torsional moments, mechanism 2 [1].

thickness direction of the RVSE extending between the two planes of symmetry of the RVSE (Figure 9(c)). Bogensperger et al. [1] adopted a simple classical torsional beam model which extends in plate thickness direction and is loaded by oppositely directed torsional moments with uniform distribution, acting antisymmetrically to the midplane of the RVSE. The assumed torsional model corresponds to the condition of restrained warping. The relevant effective shear stiffness $\left(G_{\text {eff }}\right)$ under mechanism 2 is still unknown and is assumed to be equal to $\mathrm{G}_{\text {eff, } 2}=\mathrm{G}_{\text {lam }} / 2$ (Moosbrugger et al. [19] and Bogensperger et al. [1]).

The shear deformation of mechanism $1\left(\gamma_{1}\right)$ and mechanism $2\left(\gamma_{2}\right)$ are given by (15) and (16), respectively:

$$
\begin{aligned}
& \gamma_{1}=\frac{\tau_{0}}{G_{\mathrm{lam}}}, \\
& \gamma_{2}=\frac{6 \tau_{0}}{G_{\mathrm{lam}}}\left(\frac{t}{w}\right)^{2},
\end{aligned}
$$

where $\tau_{0}$ is the nominal shear stress associated with the RVSE. $G_{\text {lam }}$ is the mean shear modulus of the laminates of the CLT parallel to grain. $t$ is the mean thickness of the laminates, and $w$ is the mean laminate width or the mean distance between stress relief cuts of CLT. As discussed previously, only a simple torsional behaviour was assumed to derive (16). The torsional shear deformation of this simple torsional beam model is given by

$$
\gamma_{2}=\frac{T L}{G_{\text {eff }, 2 J}},
$$

where $T$ is the torsional moment acting on the two sides of the RVSE at the two planes of symmetry (two outer sides). $L$ is the length from the midplane of the RVSE to the plane of symmetry. $G_{\text {eff, } 2}$ is the effective shear modulus under mechanism 2, and $J$ is the polar moment of inertia. These terms are given by the following expressions according to Bogensperger et al. [1]:

$$
\begin{aligned}
T & =\tau_{0} t a^{2}, \\
L & =\frac{t}{2}, \\
G_{\text {eff }, 2} & =\frac{G_{\text {lam }}}{2}, \\
J & =\frac{w^{4}}{6} .
\end{aligned}
$$

The torsional moment results from the nominal shear force, $\tau_{0} \cdot t \cdot w$, and $w$ is the torsional moment arm of the nominal shear force. The length from the midplane of the RVSE to the plane of symmetry is $t / 2 . G_{\text {lam }} / 2$ is the assumed value of $G_{\text {eff, } 2}$. Finally, considering the simple torsional beam model, the torsional constant is assumed to be equal to the polar moment of inertia, $J$ which for a square cross-section is given by $w^{4} / 6$. Inputting these expressions into (17) will lead to 16 .

Now, adding the shear deformation of the two mechanisms will give the total shear deformation of the CLT, which is

$$
\gamma=\gamma_{1}+\gamma_{2} \text {. }
$$

If $G$ is the effective shear modulus of the whole CLT with the superposition of both the mechanisms,

$$
\begin{aligned}
\frac{\gamma_{1}}{\gamma} & =\frac{\left(\tau_{0} / G_{\mathrm{lam}}\right)}{\left(\tau_{0} / G\right)}, \\
& \text { or } \frac{\left(\tau_{0} / G_{\mathrm{lam}}\right)}{\left(\tau_{0} / G_{\mathrm{lam}}\right)+\left(6 \tau_{0} / G_{\mathrm{lam}}\right)(t / w)^{2}} \\
& =\frac{G}{G_{\mathrm{lam}}} \\
& =\frac{1}{1+6(t / w)^{2}} \\
& \text { or } G=\frac{G_{\mathrm{lam}}}{1+6(t / w)^{2}} .
\end{aligned}
$$

Equation (20) provides a simple relation between the effective shear modulus of the CLT under in-plane loading condition and the mean shear modulus of the laminates that make up the CLT. However, as some assumptions were made regarding the shear deformation under mechanism 2 and as it can only be approximated with equation (20), Bogensperger et al. [1] carried out a finite element study for achieving better mechanical results. Also, equation (20) does not recognize the effect of the finite number of layers of CLT in predicting the shear modulus from the shear mechanism of RVSE. Instead, equation (20) assumes an infinite number of layers of the CLT. According to Turesson et al. [8], this results in an overestimate of the actual shear modulus of the CLT. The effect of the finite number of layers on equation (20) was investigated by Silly [20], and in order to account for the finite number of layers in a CLT, a correction factor 


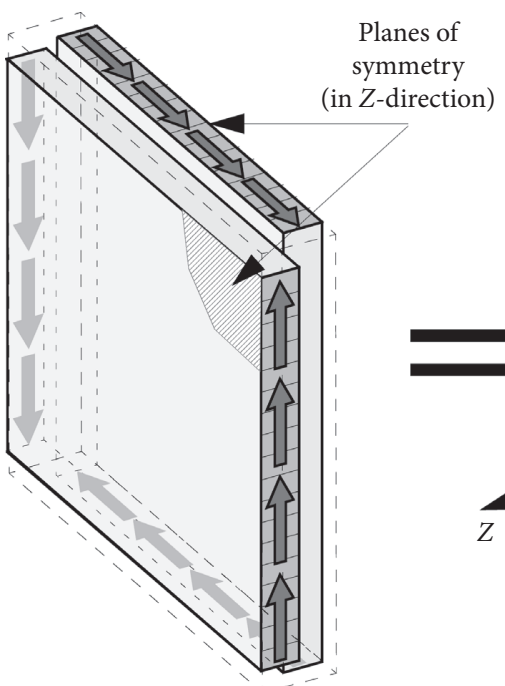

(a)

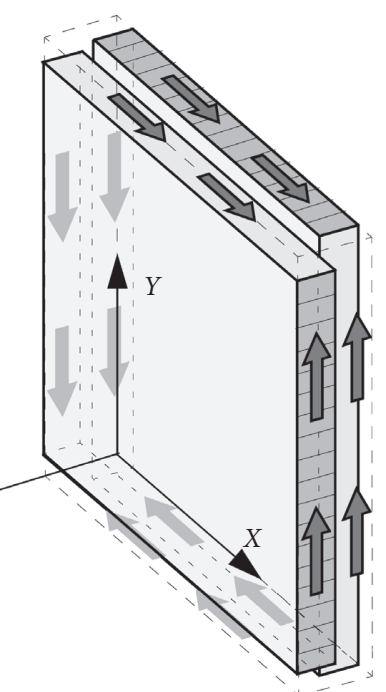

(b)

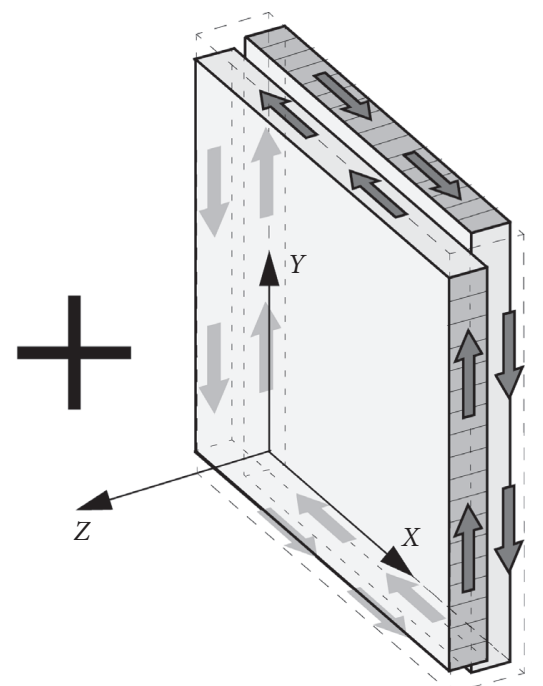

(c)

FIGURE 9: Superposition of shear mechanisms (a) original situation, (b) partial state of pure shear mechanism (mechanism 1), and (c) partial state of torsion-like behaviour (mechanism 2) [19].

was proposed. The proposed correction factor $\left(\alpha_{T}\right)$ to predict the effective shear modulus is

$$
\begin{aligned}
\alpha_{T} & =p\left(\frac{t}{w}\right)^{q} ; \\
t & =\frac{\text { total thickness of CLT }}{\text { number of layers in CLT }}
\end{aligned}
$$

where $t$ is the mean thickness of all the layers of CLT and $w$ is the laminate width of the CLT (usually a constant value for each type of CLT). The values of $p$ and $q$ in estimating the correction factor are given in Table 2 according to Silly [20] and Bogensperger et al. [1].

With the correction factor, the shear modulus is approximated by

$$
G=\frac{G_{\mathrm{lam}}}{1+6 \alpha_{T}((t / w))^{2}}
$$

Equation (22) represents the final form of the Bogensperger et al. [1] model to predict the shear modulus of CLT. Bogensperger et al. [1] assumed a mean value of the width and the thickness of the laminates in developing the model to predict the shear modulus of CLT. Though CLT with unequal width of the laminates of each layer is not common, the assumption of an equal mean thickness of all the layers means that the method cannot be applied to CLT with unequal layer thicknesses. Also, the simple torsional beam model for the shear mechanism 2 in (17) does not represent the correct state of the torsion in the RVSE when the widthto-thickness ratio of each layer is low [8]. A more accurate representation of the shear state in the RVSE can be made using the theory of elasticity.

2.4. Andreolli Method. Andreolli et al. [9] noted some of the shortcomings of the different methods mentioned in the European standard EN 16351 [21] to determine the in-plane
TABLE 2: $p$ and $q$ values of the correction factor for determining shear modulus.

\begin{tabular}{lccc}
\hline No. of layers & 3 & 5 & 7 \\
\hline$p$ & 0.53 & 0.43 & 0.39 \\
$q$ & -0.79 & -0.79 & -0.79 \\
\hline
\end{tabular}

characteristics of CLT. Among these methods, the fourpoint bending test is not always useful in determining shear properties as bending stresses are predominant compared to the shear stresses in the bending test. Other test methods conducted on small-sized specimens focus on testing of single nodes of CLT (with only one glued crossing area between the longitudinal and the transverse layers). Tests on single nodes are usually done to determine the shear strength values under different failure modes. However, they cannot provide any information on the stiffness of CLT and it is uncertain whether they are representative of the global behaviour of the whole CLT panel.

Considering the shortcomings of the previously proposed test methods, Andreolli et al. [9] proposed a diagonal compression test setup to determine some of the in-plane properties of CLT. The test setup is illustrated in Figure 10. For this test, square-shaped CLT specimen of $1 \mathrm{~m}$ side length was used. The load was applied under displacement control at a constant rate of $0.04 \mathrm{~mm} / \mathrm{s}$. The load was transferred to the specimen using reinforced steel angles (seen in the upper and lower corner of the specimen in Figure 10). The deformation of the specimen was measured on both the side faces. A total of eight instruments were positioned on the panel as shown in Figure 10. Four LVDTs measured the horizontal $\left(w_{x}\right)$ and vertical $\left(w_{y}\right)$ deformations of the central core of the specimen (the central core is the square zone located in the centre of the specimen with a diagonal length equal to $40 \%$ of the specimen diagonal, shown in Figure 10 with dashed lines), two wire potentiometers measured the 


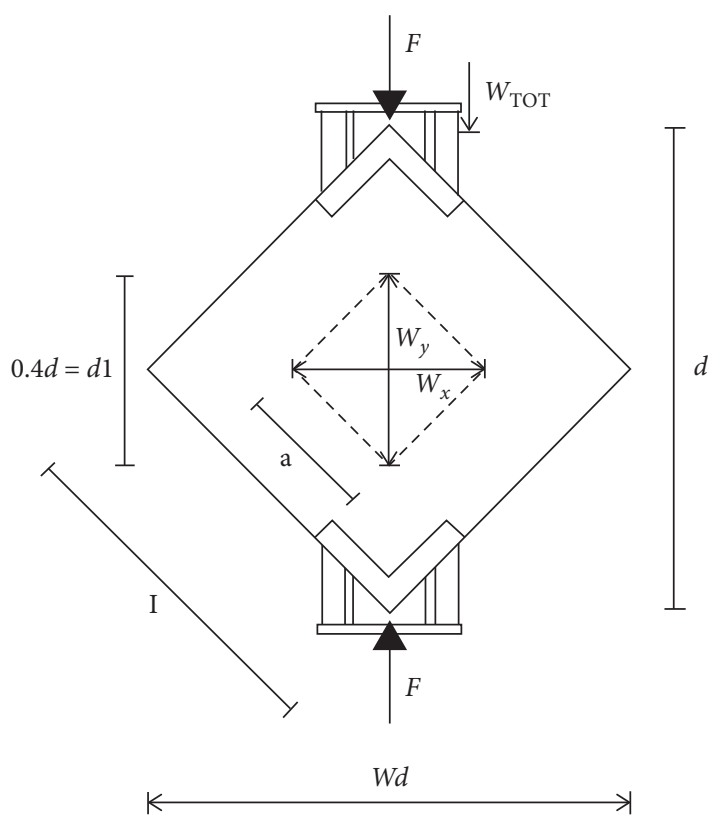

FIgURE 10: Diagonal compression test setup [8].

total deformation along the horizontal diagonals $\left(w_{d}\right)$ and two inductive displacement transducers measured the absolute vertical displacements $\left(w_{\text {Тот }}\right)$ of the machine head. A load cell was used to measure the applied force. The main goal of the diagonal compression test is to introduce a state of pure shear stress inside the CLT. For simplified treatment of the stress condition inside the CLT under diagonal compression, uniform stress distribution can be assumed. However, the actual stress distribution inside is more complex. The state of uniformity of stress is grossly violated, and in the central core zone, the shear stress is higher than other parts of the CLT.

Andreolli et al. [9] used Frocht's [22] solution to estimate the average value of the shear stress acting in the core zone from the assumed uniform stress distribution, which is given by

$$
\begin{aligned}
\tau_{\text {core }} & =1.429 \bar{\tau} \\
& =\frac{1.429 F}{\sqrt{2} \mathrm{~A}},
\end{aligned}
$$

where $F$ is the applied vertical force from the machine actuator and $A$ is the cross-sectional area of the specimen in the direction parallel to the four sides of the specimen. Thus, $A=l \times t_{\mathrm{CLT}}$, where $l$ is the length of the specimen as seen in Figure 10 and $t_{C L T}$ is the thickness of the specimen. The factor 1.429 in (23) accounts for the fact that the shear stress inside the central core zone is higher than the shear stress occurring outside the central core zone. The stress calculated using $F / \sqrt{2} A$ in (23) gives the value of the stress if uniform stress distribution is considered throughout the CLT. Andreolli et al. [9] suggested this factor by averaging the ratios of the actual the shear stress in the core zone to the shear stress calculated using $F / \sqrt{2} A$ along the horizontal (along $w_{x}$ ) and vertical (along $w_{y}$ ) diagonal of the central core zone.
The average shear strain of the core zone is given by

$$
\gamma=\frac{2 \Delta v}{d_{1}}
$$

The derivation of (24) can be explained with the help of the deformed shape of the core zone (Figure 11).

$\Delta v$ in (24) is the average change in the core diagonal of length $d_{1}$ as measured by the four LVDT transducers, thus $\Delta v=\left(w_{x, \text { side } 1}+w_{x, \text { side } 2}+w_{y \text {,side } 1}+w_{y, \text { side } 2}\right) / 4$. In Figure 11, the deformed shape is shown with dotted lines. $a$ is the length of the core zone. From small-displacement approximations,

$$
\begin{aligned}
\cos (90-\gamma)=\sin \gamma & \approx \gamma, \\
\Delta v^{2} & \approx 0 .
\end{aligned}
$$

Using cosine law of triangle,

$$
\begin{aligned}
d_{1}-\Delta v & =\sqrt{a^{2}+a^{2}-2 \cdot a \cdot a \cdot \cos (90-\gamma)}, \\
\text { or }\left(d_{1}-\Delta v\right)^{2} & =a^{2}+a^{2}-2 \cdot a \cdot a \cdot \cos (90-\gamma) \\
\text { or } d_{1}^{2}+\Delta v^{2}-2 d_{1} \Delta v & =a^{2}+a^{2}-2 \cdot a \cdot a \cdot \cos (90-\gamma) \\
\text { or }-2 d_{1} \Delta v & =-d_{1}^{2} \gamma \\
{\left[\Delta v^{2}\right.} & \approx 0 \text { and from the undeformed shape, } \\
d_{1}^{2} & \left.=a^{2}+a^{2}\right] \\
\text { or } \gamma & =\frac{2 \Delta v}{d_{1}} .
\end{aligned}
$$

Finally using (23) and (24), the shear stress versus shear strain curve of the core zone is produced for each specimen. Regression analysis is carried out on the data points to get a best-fit line. Then, the slope of the best-fit line within the $10 \%-40 \%$ range (this range is actuated following the provisions of EN 408 [11]) of the maximum applied load $F_{\max }$ gives the value of the in-plane shear modulus of CLT, G.

Andreolli et al. [9] conducted diagonal compression test on four types of CLTs: (1) CLT with narrow face bonding; (2) CLT with narrow face bonding but with shrinkage cracks; (3) CLT without narrow face bonding and with stress relief cuts; (4) CLT without narrow face bonding. The first type of CLT gave the highest value of shear modulus, which was comparable to that of solid lumber material.

Turesson et al. [23] discussed some of the drawbacks of the diagonal compression test. The contact area between the CLT panel and the steel angles (which act as supports) provides some additional restraint to the deformation of the CLT in the horizontal direction. As a result, the CLT panels are not deformed in the vertical and horizontal direction by the same amount. The factor 1.429 used to calculate the shear stress in the core zone does not consider this unequal deformation issue. Furthermore, the simplified deformed shape exhibited in Figure 11, in which both the vertical and 


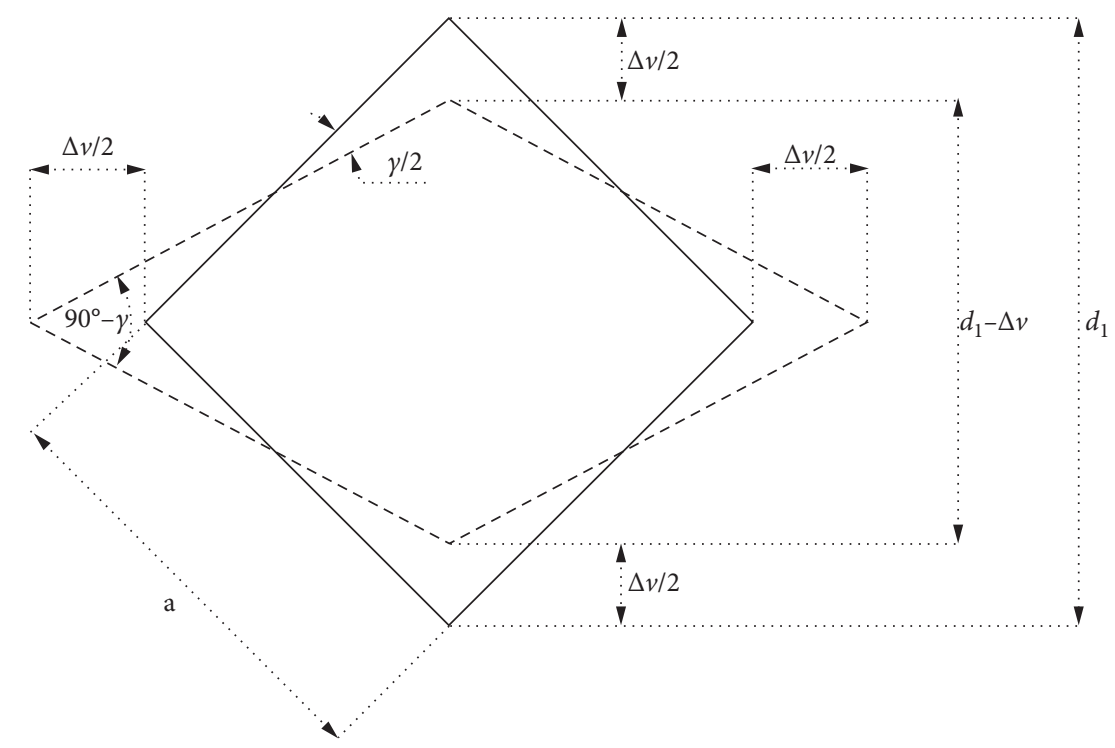

FIGURE 11: The central core zone in the undeformed (represented by solid lines) and deformed (represented by dashed lines) shape.

horizontal deformation is assumed to be equal $(\Delta v / 2)$, is not representative of the actual deformed shape of the core zone due to the unequal deformation in the vertical and horizontal direction. This might lead to inaccurate value of the shear modulus determined using Andreolli et al.'s [9] method based on the geometry of the deformed shape. Considering this shortcoming, Turesson et al. [23] developed a picture frame test setup, which is essentially an improvement over that used by Andreolli et al. [9]. According to Turesson et al. [23], CLT panels can be deformed along the horizontal and vertical diagonals proportionately by the same amount using the picture frame setup, which was not the case for specimen in the diagonal compression test of Andreolli et al. [9]. However, the picture frame test setup by Turesson et al. [23] will not be described here as the principle is the same as that of Andreolli et al. [9].

2.5. Turesson Method. Turesson et al. [8] carried out finite element analysis to derive some reduction factors, which when multiplied by the shear modulus value of the lumber forming the CLT (assuming each layer of CLT is formed with the same grade of lumber), will give the effective shear modulus value of the whole CLT. The reduction factor $\left(k_{88}\right)$ ranges from a value of 0 to 1 . According to Turesson et al. [8], its value is 1 for an edge-glued CLT and for a non-edge-glued CLT, it is always less than 1. While Bogensperger et al. [1] only analysed idealised representative volume subelements of CLT as discussed previously, Turesson et al. [8] analysed whole sized $(1.28 \mathrm{~m} \times 1.28 \mathrm{~m})$ 3-layered and 5-layered CLT panels using finite element methods.

In their study, Turesson et al. [8] modelled the whole CLT panels and analysed them using proper boundary conditions by finite element analysis. Shear force was applied on the sides of the panels as surface traction forces. A simplified model of the CLT panel is shown in Figure 12. Two corner nodes were restrained against translation in the vertical and horizontal direction to prevent any rigid body motion of the panel. The

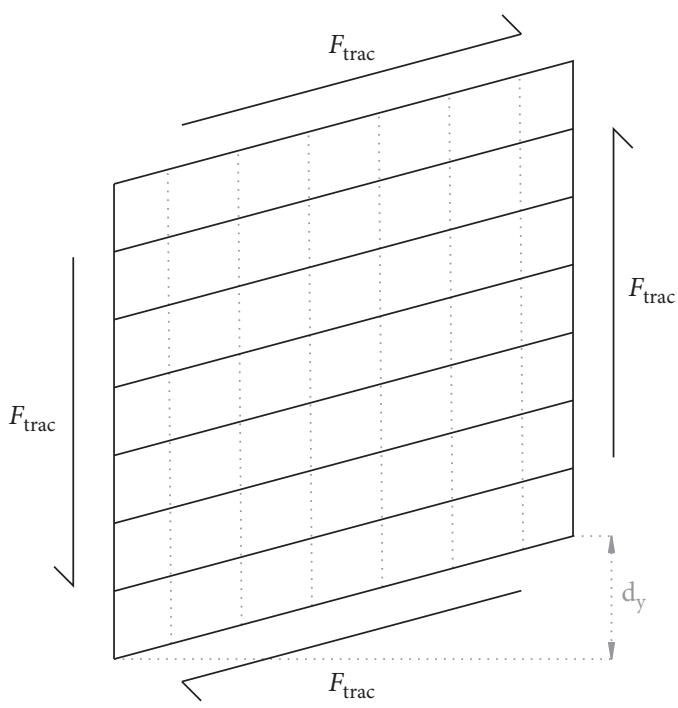

FIgURE 12: Finite element model of the CLT panel. The reduction factor was calculated using equation (27).

two corners on the left edge of the panel were restrained from any movement. The right edge of the panel was shifted by an amount of $d_{y}$ under the action of shear force:

$$
k_{88}=\frac{F_{\mathrm{trac}}}{t_{\mathrm{CLT}} d_{y} G_{\mathrm{lam}}},
$$

where $F_{\text {trac }}$ is the applied traction force in the finite element analysis software, $t_{\text {CLT }}$ is the total thickness of the CLT, $d_{y}$ is the displacement of the CLT due to the shear force, and $G_{\text {lam }}$ is the mean shear modulus value of the laminates of the CLT.

As reflected in (27), the reduction factors were calculated relative to the shear modulus of the laminates of the CLT, $G_{\text {lam }}$. From the finite element analysis, Turesson et al. [8] generated charts for determining the value of the factor based on width of the laminates and thickness of each layer. 
From the large range of values of the width of the laminates and thickness of the layers, practical values used in real-life CLTs can easily be determined from the charts. Turesson et al. [8] also suggested an equation to predict the correction factors based on curve fitting methods. Nevertheless, Turesson et al. [8] only studied 3- and 5-layer CLT panels, and the results cannot be extended to CLT panels with a greater number of layers.

2.6. Comparison of the Methods and Analysis of the Results. Unlike the Flaig and Blaß [5] method, Brandner et al.'s [6] method requires test results for each specific type of CLT as the $E_{y}$ value will vary depending on the type of CLT. In other words, the Brandner et al. [6] model to predict the shear modulus of CLT might be considered a semiempirical approach while the Flaig and Blaß [5] model is more of a mechanics-based approach. Large-scale tests on CLT panels were carried out by Zimmerman and McDonnell [24] to determine the effective in-plane shear modulus of full-size CLT panels. The effective shear modulus values were compared against the values predicted by Flaig and Blaß [5] and Brandner et al. [6] models. From the comparison, it was observed that both methods gave underestimated values of the effective shear modulus compared to the test results. However, the values predicted by the Flaig and Blaß [5] method were further off from the test results compared to the values predicted by Brandner et al.'s [6] method. It should be noted that CLTs in the large-scale panel tests were under constant axial compression during testing which might have contributed to relatively higher effective shear modulus values. Zimmerman and McDonnell [24] also reported that the predicted effective shear moduli using these Flaig and Blaß [5] and Brandner et al. [6] methods differed by approximately a factor of two.

Flaig and Blaß [5] provided a plot of the effective shear modulus of CLT calculated using (14) with a shear modulus value of $690 \mathrm{MPa}$ for the laminates and a slip modulus $(K)$ value of $5 \mathrm{~N} / \mathrm{mm}^{3}$. The width of the laminates $(w)$ has a big influence on the shear modulus value. As discussed previously, the shear modulus predicted by the Flaig and Blaß [5] method is dependent on the number of adjacent laminates in the longitudinal layers $(m)$. However, for CLTs with the same width of the laminates, with an increasing number of laminates in the longitudinal layers $(m)$, the difference in the predicted shear modulus values gradually decreases. The shear modulus value can be taken as constant if the number of laminates in the longitudinal layers is equal to or exceeds 10. In Figure 13, the shear modulus values of CLT predicted by the Flaig and Blaß [5] method with $G_{\text {lam }}=690 \mathrm{MPa}$, $K=5 \mathrm{~N} / \mathrm{mm}^{3}$, and $m=10$ have been compared with the shear modulus determined by Andreolli et al. [9] and Brandner et al. [6] methods for CLT with comparable physical properties. It can be observed that the Flaig and Blaß [5] method underestimates the shear modulus value compared to the other two methods which corroborate the findings of Zimmerman and McDonnell [24]. A possible reason for the underestimated values of shear modulus by the Flaig and Blaß [5] method is the use of an inappropriate

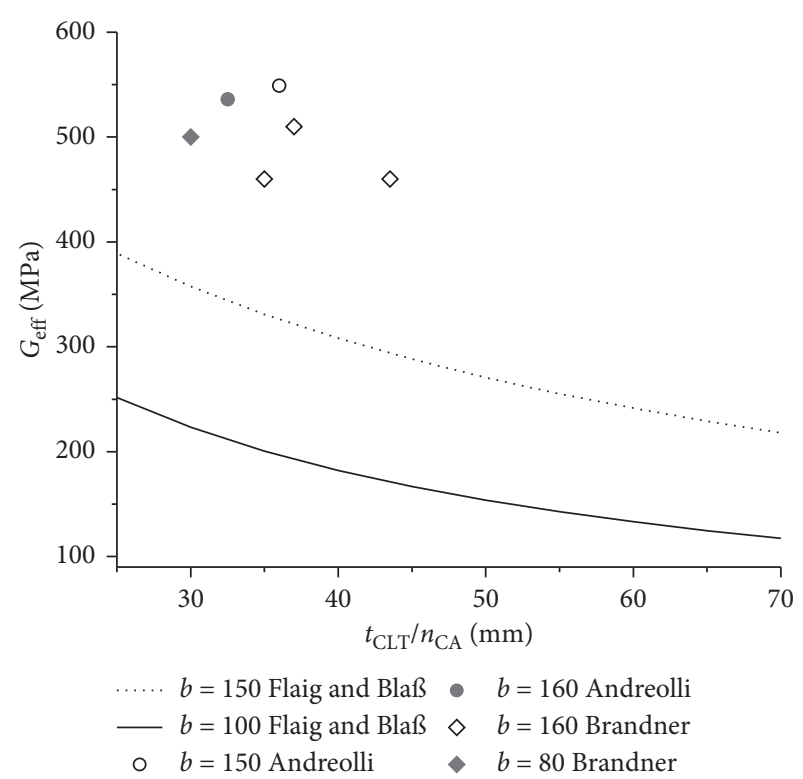

Figure 13: Effective shear modulus vs. $t_{\mathrm{CLT}} / n_{\mathrm{CA}}$ for CLT. The laminate width $(b)$ is in $\mathrm{mm}$.

value of $K$. As discussed by Blaß and Flaig [13], the determination of an appropriate value of $K$ poses a challenge in reality.

Turesson et al. [8] suggested some shear modulus values of CLT using finite element analysis and compared these values to the experimental values of Brander at al. [6]. The difference in the values was between $0.4 \%$ and $6.9 \%$. Brandner et al. [5] compared the shear modulus values determined from their compression tests to those predicted using the mechanical model given by Bogensperger et al. [1]. Brandner et al. [5] reported that the shear modulus values determined from the two methods were overall coherent, except for some CLTs with stress reliefs. For reducing the effects of shrinkage-swelling stresses and subsequent formation of cracks with the change of surrounding moisture content in CLT panels, stress relief was introduced by making cuts in the longitudinal laminates of the CLT panels [25]. A detailed description of CLT stress relief cut can be found in the CLT Handbook [26]. In a practical scenario, in CLTs with stress reliefs, some additional stiffness is developed due to (i) the residual bridge at the groove of the relief (the bridge occurs as the grooves are not always such that the cuts extend the full thickness of each layer) and (ii) unintentional penetration of the adhesive into the reliefs. In Figure 14, the effective shear modulus value of CLT as a fraction of the shear modulus of the laminates of the CLT determined by the methods proposed by Bogensperger et al. [1], Brandner et al. [6], Turesson et al. [8] and Andreolli et al. [9] has been plotted against the mean layer thickness-towidth of laminates ratio for CLTs with a different number of layers. Here, $t_{\text {mean }}$ is the total thickness of the CLT divided by the number of layers and $w$ is the width of the laminates of the CLT. For the shear modulus values from Andreolli et al. [9], the qualifier "equal" represents an equal thickness of all layers, "unequal" represents an unequal thickness of all 


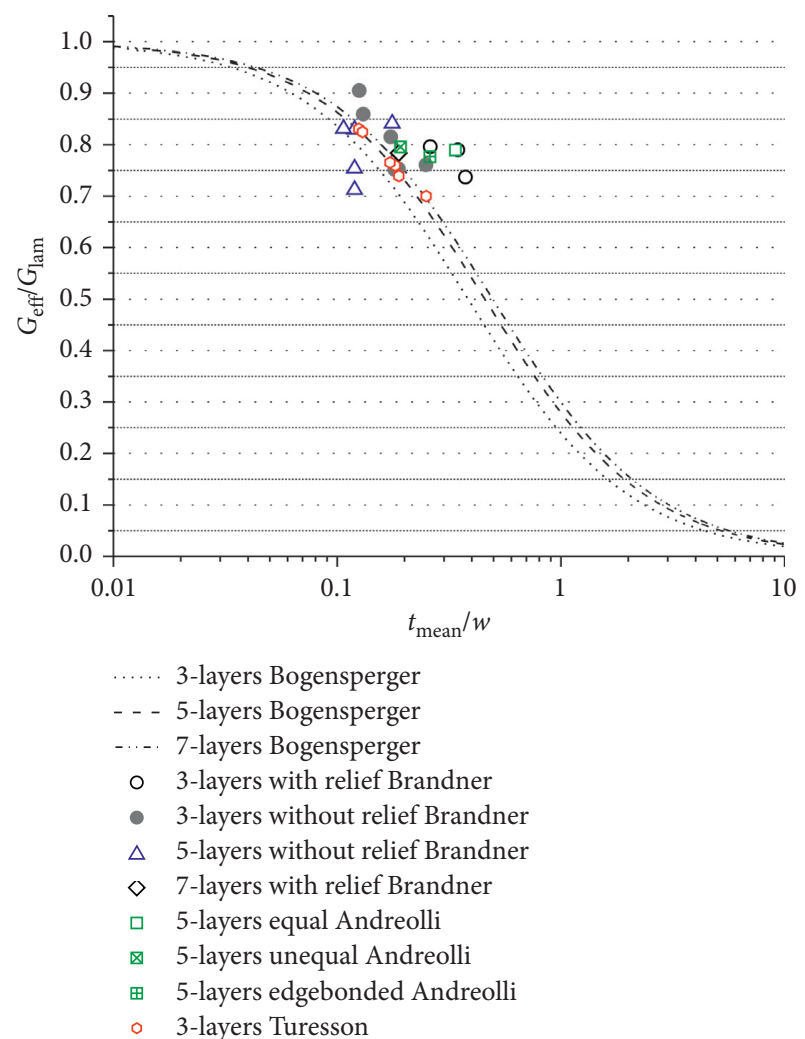

FIgURE 14: Effective shear modulus of CLT vs. geometric ratio $t_{\text {mean }} / w$.

layers, and "edgebonded" represents an edge-glued CLT. The shear modulus of an edge-glued CLT with the presence of shrinkage cracks from the study of Andreolli et al. [9] has been presented along with the shear modulus of all other non-edge-glued CLTs in Figure 14. The shear modulus value of the edge-glued CLT is similar to those of the non-edgeglued CLTs. The reason for this is the edge-glued CLT in Andreolli et al.'s [9] study had developed considerable shrinkage cracks, which eventually happens in all edge-glued CLTs in real life due to the effect of moisture-induced stresses caused by the change in surrounding moisture content. After the formation of shrinkage cracks, the behaviour of an edge-glued CLT becomes closer to that of a non-edge-glued CLT and it no longer exhibits high shear modulus values concordant with that of solid lumber.

From Figure 14, it can be observed that the shear modulus values from all the four methods are closely clustered and Brandner et al. [6] and Andreolli et al. [9] methods give slightly overestimated shear modulus values compared to Bogensperger et al.'s [1] method. Turesson et al.'s [8] method gives shear modulus values very close to those from Bogensperger et al.'s [1] method.

Turesson et al. [23] reported that Brandner et al.'s [6] method is increasingly becoming accepted as the most appropriate test setup for determining the gross shear properties of CLT. However, ensuring a pure shear stress condition remains a problem in this method. The Flaig and $\mathrm{Blaß}$ [5] method is very convenient as it does not require any physical testing and only the material properties of the CLT are required as input parameters. The Flaig and Blaß [5] method has the potential to become widely accepted if the relevant shear stiffness of the crossing areas under the two separate mechanisms can be properly quantified. Bogensperger et al. [1] and Turesson et al. [8] methods are mainly dependent on numerical modelling and simulation of CLT using finite element methods. As it is the case for all finite element methods, some simplified approximations are always present. For example, modelling the glue layer alone poses a challenge and the results can be drastically different depending on the method used to model the glue layer in the finite element analysis software. Andreolli et al.'s [9] method is also a very good experimental method for determining the shear modulus, but the test setup implementation remains a problem due to the large sample sizes and the requirement of fabricating special fixtures. Thus, all the methods described in this paper have some pros and cons. Each of these methods has room for improvement through further research.

\section{Conclusions}

Five methods for the determination of the in-plane shear modulus of CLT have been discussed in this paper. Each method has some advantages and disadvantages. Some of them require physical testing, while others are just prediction models that require the material properties of CLT as input parameters. The highlights of the five methods are as follows:

(1) The Brandner method is a semiempirical approach which gives shear modulus values close to the values from large-scale CLT panel tests. The size of the specimens used in this method is fairly large, which is cumbersome.

(2) The Flaig and Blaß method is a mechanics-based analytical approach. However, determination of one of the input properties of the analytical model and the stiffness of the crossing areas remains a challenge.

(3) The Bogensperger method is a combination of analytical and numerical approach. The analytical part of this method relies on some simplified assumptions, which might not be very accurate.

(4) The Andreolli method involves experimental determination of the shear modulus from a diagonal compression test. The geometric assumptions made in this method might not be appropriate due to the nonuniform deformation in the vertical and horizontal direction.

(5) In the Turesson method, shear modulus was found from numerical modelling. A prediction model was proposed from numerical analysis; however, the model is valid for only 3-and 5-layered CLT panels.

Further research is needed to develop a method that can accurately predict the shear modulus of CLT and that takes into account the shortcomings of all the current methods. Thus, the full potential of CLT can be utilised. 


\section{Data Availability}

Most of the data have been taken from other literature studies as this is a review paper. However, the authors of this paper have reproduced all the graphs used in this paper from different sources. All the reproduced data used in this paper are available upon request from the authors of this paper.

\section{Conflicts of Interest}

The authors declare that there are no conflicts of interest regarding the publication of this paper.

\section{Acknowledgments}

Financial support to this research was provided by the Natural Sciences and Engineering Research Council of Canada (NSERC) Industrial Research Chair grant program and industrial partners: FPInnovations, Western Archrib, Landmark Group, Canadian Wood Council, ACQBuild, MTC Solutions, and Rotho Blaas. Test material was provided by Nordic Structures. Their contributions are gratefully acknowledged.

\section{References}

[1] T. Bogensperger, T. Moosbrugger, and G. Silly, "Verification of CLT-plates under loads in plane," in Proceedings of the 11th World Conference on Timber Engineering, Riva del Garda, Italy, June 2010.

[2] M. Shahnewaz, M. S. Alam, T. Tannert, and M. Popovski, "Cross laminated timber walls with openings: in-plane stiffness prediction and sensitivity analysis," in Proceedings of the Annual Conference of the Canadian Society for Civil Engineering, London, UK, June 2016.

[3] H. Ferk, "Some building science aspects for building with CLT," in Proceedings of the European Conference on Cross Laminated Timber (CLT)-Focus Solid Timber Solutions, pp. 207-250, Bath, UK, 2013.

[4] M. Jeleč, D. Varevac, and V. Rajčić, "Cross-laminated timber (CLT) - a state of the art report," Gradjevinar, vol. 70, 2018.

[5] M. Flaig and H. J. Blaß, "Shear strength and shear stiffness of CLT-beams loaded in plane," in Proceedings of the CIB 2013, Vancouver, Canada, May 2013.

[6] R. Brandner, P. Dietsch, J. Dröscher, M. Schulte-Wrede, H. Kreuzinger, and M. Sieder, "Cross laminated timber (CLT) diaphragms under shear: test configuration, properties and design," Construction and Building Materials, vol. 147, pp. 312-327, 2017.

[7] J. Zhou, Y. H. Chui, M. Gong, and L. Hu, "Elastic properties of full-size mass timber panels: characterization using modal testing and comparison with model predictions," Composites Part B: Engineering, vol. 112, pp. 203-212, 2017.

[8] J. Turesson, S. Berg, and M. Ekevad, "Impact of board width on in-plane shear stiffness of cross-laminated timber," Engineering Structures, vol. 196, Article ID 109249, 2019.

[9] M. Andreolli, M. A. Rigamonti, and R. Tomasi, "Diagonal compression test on cross laminated timber panels," in Proceedings of the 13th World Conference on Timber Engineering, Quebec, Canada, August 2014.

[10] H. Kreuzinger and M. Sieder, "Einfaches Prüfverfahren zur Bewertung der Schubfestigkeit von Kreuzlagenholz/Brettsperrholz," Bautechnik, vol. 90, no. 5, pp. 314-331, 2013.
[11] CEN, EN 408+A1: Timber Structures-Structural Timber and Glued Laminated Timber-Determination of Some Physical and Mechanical Properties, CEN, Brussels, Belgium, 2010.

[12] M. Flaig and N. Meyer, "A new test configuration to determine the slip modulus of connections between crosswise bonded boards," in Proceedings of the Experimental Research with Timber, pp. 77-84, Prague, Czech Republic, May 2014.

[13] H. J. Blaß and M. Flaig, "Stabförmige bauteile aus brettsperrholz," Karlsruher Berichte zum Ingenieurholzbau, Bd. 24, KIT Scientific Publishing, Karlsruhe, Germany, 2012.

[14] G. R. Cowper, "The shear coefficient in timoshenko's beam theory," ASME: Journal of Applied Mechanics, vol. 33, no. 2, pp. 335-340, 1966.

[15] H. J. Blaß and R. Görlacher, "Zum trag- und verformungsverhalten von brettsperrholzelementen bei beanspruchung in plattenebene," Bauen Mit Holz, vol. 104, pp. 34-41, 2002.

[16] R. A. Jöbstl, T. Bogensperger, and G. Schickhofer, "Mechanical behaviour of two orthogonally glued boards," in Proceedings of the 8th World Conference on Timber Engineering, pp. 357-364, Lahti, Finland, June 2004.

[17] CEN, EN 338: Structural Timber-Strength Classes, CEN, Brussels, Belgium, 2009.

[18] H. Danielsson and E. Serrano, "Cross laminated timber at inplane beam loading-prediction of shear stresses in crossing areas," Engineering Structures, vol. 171, pp. 921-927, 2018.

[19] T. Moosbrugger, W. Guggenberger, and T. Bogensperger, "Cross laminated timber wall segments under homogeneous shear-with and without openings," in Proceedings of the 9th World Conference on Timber Engineering, Portland, OR, USA, August 2006.

[20] G. Silly, "Numerische studien zur drill- und schubsteifigkeit von brettsperrholz (BSP)," Diploma thesis, Institute for Timber Engineering and Wood Technology, Graz University of Technology, Graz, Austria, 2010.

[21] CEN, En 16351: Timber Structures-Cross Laminated Timber-Requirements, CEN, Brussels, Belgium, 2015.

[22] M. M. Frocht, "Recent advances in photoelasticity and an investigation of the stress distribution in square blocks subjected to diagonal compression," ASME Transactions, vol. 55, pp. 135-153, 1931.

[23] J. Turesson, A. Björnfot, S. Berg et al., "Picture frame and diagonal compression testing of cross-laminated timber," Materials and Structures, vol. 52, p. 66, 2019.

[24] R. B. Zimmerman and E. Mcdonnell, "Framework-a tall recentering mass timber building in the United States," in Proceedings of the New Zealand Society for Earthquake Engineering Conference, Wellington, New Zealand, April 2017.

[25] M. He, X. Sun, Z. Li, and W. Feng, "Bending, shear, and compressive properties of three- and five-layer cross-laminated timber fabricated with black spruce," Journal of Wood Science, vol. 66, no. 1, p. 38, 2020.

[26] E. Karacabeyli and S. Gagnon, Canadian CLT Handbook, FP Innovations, Pointe-Claire, Canada, 2nd edition, 2019. 